\title{
ELSA: An integrated, semi-automated nebular abundance package
}

\author{
Matthew D. Johnson ${ }^{1}$, Jesse S. Levitt ${ }^{2}$, \\ Richard B. C. Henry ${ }^{3}$, and Karen B. Kwitter ${ }^{2}$ \\ ${ }^{1}$ Department of Astronomy, Wesleyan University, Middletown, CT 06459, USA \\ email: mdjohnson@wesleyan.edu \\ ${ }^{2}$ Department of Astronomy, Williams College, Williamstown, MA 01267, USA \\ email: 08jsl@williams.edu,kkwitter@williams.edu \\ ${ }^{3}$ H. L. Dodge Department of Physics and Astronomy, U. Oklahoma, Norman, OK 73019, USA \\ email: henry@nhn.ou.edu
}

\begin{abstract}
We present ELSA, a new modular software package, written in C, to analyze and manage spectroscopic data from emission-line objects. In addition to calculating plasma diagnostics and abundances from nebular emission lines, the software provides a number of convenient features including the ability to ingest logs produced by IRAF's splot task, to semi-automatically merge spectra in different wavelength ranges, and to automatically generate various data tables in machine-readable or $\mathrm{LT}_{\mathrm{E} X} \mathrm{X}$ format. ELSA features a highly sophisticated interstellar reddening correction scheme that takes into account temperature and density effects as well as $\mathrm{He}$ II contamination of the hydrogen Balmer lines. Abundance calculations are performed using a 5-level atom approximation with recent atomic data, based on R. Henry's ABUN program. Downloading and detailed documentation for all aspects of ELSA are available at the following URL:

http://www.williams.edu/Astronomy/research/PN/
\end{abstract}

Keywords. planetary nebulae: general, methods: data analysis, ISM: abundances

\section{Overview}

ELSA automates some of the more laborious aspects of spectroscopic data analysis for emission-line objects, and provides highly accurate and refined routines for dereddening and abundance calculations. Furthermore, it can generate output to screen, to spreadsheet delimited file formats, or to $\mathrm{IAT}_{\mathrm{E} X}$ source files for each step of the analysis.

ELSA takes the output from IRAF's splot task and matches the measured wavelengths (adjustable for a user-input radial velocity) to a list of reference wavelengths provided by the user. ELSA can then correct for interstellar reddening, generate a wavelength-flux data table, compute plasma diagnostics and elemental abundances, and flag unknown or commented wavelengths for later review. User-defined comments in the original logfiles are preserved throughout the analysis process. It is also possible to bypass the reddening correction phase in order to run analysis of data that has been previously dereddened. Future plans for ELSA include the incorporation of robust error propagation, the addition of UV and IR analyses, expansion to a multiple-zone model (similar to IRAF's nebular package) and support to accommodate extragalactic emission-line objects.

\section{Reddening Correction \& $\mathbf{T}_{e}-\mathbf{N}_{e}$ Loops}

Dereddening is performed through an iterative process that works in several stages: correcting for the $\mathrm{He}^{+2}$ Pickering line contamination of the $\mathrm{H} \alpha$ and $\mathrm{H} \beta$ lines, accounting for the co-dependence of $\mathrm{T}_{e}$ and $\mathrm{N}_{e}$ determinations, dynamically calculating the appropriate $\mathrm{H} \alpha / \mathrm{H} \beta$ ratio based on $\mathrm{T}_{e}$ and $\mathrm{N}_{e}$, and calculating the reddening coefficient $c$ based 
on the appropriate intrinsic Balmer ratio. ELSA makes a first-pass correction assuming a user-defined default $\mathrm{H} \alpha / \mathrm{H} \beta$ (e.g., 2.86). This rough value is used to apply a reddening correction from Savage \& Mathis (1979) to $\mathrm{H} \alpha$ and He II $\lambda 4686$. The corrected $\lambda 4686$ flux is then used to calculate the contributions of coincident $\mathrm{He}^{+2}$ Pickering lines to be subtracted from observed $\mathrm{H} \alpha$ and $\mathrm{H} \beta$. The reddening correction then runs inversely to "reredden" $\mathrm{H} \alpha$ and $\mathrm{H} \beta$. A new value of $c$ is then computed and the process repeats until $c$ converges. The final $c$ is used to deredden [O III $] \lambda 4363$ and $\lambda 5007$, which are used to derive $\mathrm{T}_{e}$ assuming a fixed "seed" $\mathrm{N}_{e}$. A $2 \mathrm{D}$ polynomial interpolation table is used to determine $\mathrm{N}_{e}$ using the previously calculated $\mathrm{T}_{e}$. A new $\mathrm{T}_{e}$ is then calculated based on the improved $\mathrm{N}_{e}$, and so on until both approach stable values.

The $\mathrm{T}_{e}$ and $\mathrm{N}_{e}$ are used to refine the intrinsic $\mathrm{H} \alpha / \mathrm{H} \beta$ ratio, using data and code based on Storey \& Hummer (1995). ELSA then returns to the beginning of the loop to make a second pass at dereddening the raw data with the new $\mathrm{H} \alpha / \mathrm{H} \beta$ used to determine $c$. The entire loop iterates in this manner until $\mathrm{H} \alpha / \mathrm{H} \beta$ itself converges satisfactorily. The final $c$ is then used to deredden all raw line data. ELSA then stores this as the pool of data to be used for generating tables or computing chemical abundances.

\section{Chemical Abundance Calculations}

ELSA uses a standard five-level atom model as described in Kwitter \& Henry (2001), with code based on Henry's ABUN routine. ELSA can compute abundances for various ionization stages of $\mathrm{He}, \mathrm{N}, \mathrm{O}, \mathrm{S}, \mathrm{Ar}$ and $\mathrm{Ne}$; total abundances are computed using ionization correction factors as described in Kwitter \& Henry (2001). Atomic data are drawn principally from Osterbrock \& Ferland (2005) or from Mendoza (1983). Currently, ELSA contains a two-zone ionization model, with $\mathrm{T}_{[N I I]}$ and $\mathrm{T}_{[O I I I]}$ regions.

\section{Tabular Output Options}

ELSA can produce output in variety of formats. The dereddened line fluxes can be output to a tab-delimited file, a comma-separated value (CSV) file readable by spreadsheet programs such as Microsoft Excel, or a $\mathrm{LAT}_{\mathrm{E}} \mathrm{X}$ source file suitable for publication. The content of this table is controlled by a user-defined list of wavelengths, and corrected line intensities are given relative to $\mathrm{H} \beta=100$. ELSA also has the capability to produce a composite table of the same lines across multiple objects in $\mathrm{H}_{\mathrm{A}} \mathrm{T}_{\mathrm{E}} \mathrm{X}$ format, a product that would otherwise be quite laborious to produce. Comments read from log files are also preserved and output into the tabular results.

\section{Acknowledgements}

We are grateful to the Keck Northeast Astronomy Consortium, which is partially supported by the National Science Foundation, and to the Bronfman Science Center and Astronomy Department of Williams College. We also acknowledge National Science Foundation grant AST 03-07118 to the University of Oklahoma.

\section{References}

Kwitter, K.B., \& Henry, R.B.C. 2001, ApJ, 562, 804

Mendoza, C. 1983, MNRAS, 202, 981

Osterbrock, D. E., \& Ferland, G.J. 2005, Astrophysics of Gaseous Nebulae and Active Galactic Nuclei, $2^{\text {nd }}$ edition, University Science Books, Sausalito, CA, USA

Savage, M.J., \& Mathis, J. S. 1979, ARA $\mathscr{G} A, 17,73$

Storey, P.J., \& Hummer, D.G. 1995, MNRAS, 272, 41 